\section{Quick-Reference Protocol Manual for Nuclear Medicine Technologists}

\author{
M.B. Farrell, Ed.
}

Reston, VA: Society of Nuclear Medicine and Molecular Imaging, 2014, 344 pages, $\$ 83.95$

The new publication Quick-Reference Protocol Manual for $\mathrm{Nu}$ clear Medicine Technologists, published by the Society of Nuclear Medicine and Molecular Imaging (SNMMI) in 2014, is a great addition to our field. Five well-respected nuclear medicine technologists are listed as the authors, and they acknowledge several physicians and technologists who assisted with expertise and review. The preface notes that the book is intended to serve as a reference source that complements (but does not substitute for) published guidelines or a departmental protocol manual. I believe it accomplishes this purpose well.

The introduction addresses steps common to many studies, including the initiation of the procedure, administration of the radiopharmaceutical, and image acquisition. It has a helpful section on working with children. Each section has multiple subsections. These are up to date in regard to recent health-care trends such as privacy and confidentiality, appropriate use, and dose reduction. Although the book as a whole is designed to provide specific suggestions, the introduction makes it clear that technologists must be flexible in adapting protocols to individual situations. As an educator, I will make good use of this introductory chapter with my students as they enter the clinical setting.

The remainder of the book discusses 62 diagnostic and 9 therapeutic nuclear medicine procedures. Each has subsections addressing the following topics: rationale and introduction, indications and contraindications, patient preparation, radiopharmaceuticals and dosages, acquisition parameters and options, and processing instructions. The rationale and introduction subsection is a short paragraph, whereas the other subsections are organized as numbered lists, making it easy to find the information one needs. The acquisition parameters section includes tables listing standard and optional choices; when appropriate, separate tables are presented for planar and SPECT imaging. Hybrid options including CT are listed, but CT acquisition parameters are not; the reader is referred to manufacturers' protocols. Administration protocols are given in tabular form as well.

The diagnostic studies are grouped by organ system or study type (infection and inflammation, cell labeling, oncology), and this organization extends to the edge of each page, making it easy to find a given section of the book. The header on each page also references the organ system; I would have preferred to have the individual study at the top of the right-hand page.
Although I have other minor quibbles with organization (I would have separated thyroid uptake from thyroid imaging, and hyperthyroid therapy from thyroid cancer therapy), overall I find the book to be well thought out in terms of its layout.

An especially helpful aspect of the book is its description of pharmacologic agents. Stress pharmaceuticals for myocardial perfusion are given their own sections, with subsections on indications, contraindications, patient preparation, dose and infusion protocols, procedure, reasons for early termination or reversal, treatment of adverse reactions, and adjunct or interventions. Having this important information available in such an accessible format is a great asset in and of itself. Exercise stress testing is also given its own section with similar subsections, and it helpfully lists generic and trade names of common cardiac medications that interfere with exercise testing. Other pharmacologic agents are discussed in the context of their applicable procedures.

My only real concern about this reference book is the fact that it is bound. As the authors note in the preface, nuclear medicine is a dynamic imaging modality. Today's recommendations may be superseded in a fairly short time, as new radiopharmaceuticals become available and recommended protocols change. I would love to see this published in a way that allows for changes and updates, perhaps in a binder format with new pages becoming available to original purchasers or via an ongoing subscription of some fashion. On the other hand, the bound version is small enough $(15 \times 23 \mathrm{~cm}[6 \times 9 \mathrm{in}])$ to be easily portable. And the fact that it is bound decreases the likelihood that pages will be lost, always a problem with binder-type formats.

Either way, I think the book would make an excellent addition to every nuclear medicine department. It would be extremely useful in reviewing protocols and exploring alternate methods to accomplish different studies. And it would be invaluable when that rarely performed examination is requested. I commend the authors and the SNMMI for addressing the need for high-quality reference materials in our field. The strongest endorsement I can give is from one of my students, who says she uses it on a daily basis.

\author{
Jennifer Prekeges \\ Bellevue College \\ 3000 Landerholm Circle SE \\ Bellevue, WA 98007-6484 \\ E-mail: jennifer.prekeges@bellevuecollege.edu
}

Published online Jun. 26, 2014. DOI: $10.2967 /$ jnmt.114.143610 